\title{
Tracheal Stenosis: A Case Report
}

\author{
Jefri Adi Kam Sitepu' ${ }^{1}$, Marjono Dwi Wibowo ${ }^{2}$ \\ ${ }^{1}$ Department of Surgery, Faculty of Medicine Universitas Airlangga \\ Dr. Soetomo General, Hospital, Surabaya, Indonesia \\ ${ }^{2}$ Division of Head and Neck Surgery, Department of Surgery, Faculty of Medicine \\ Universitas Airlangga/ Dr. Soetomo General Hospital, Surabaya, Indonesia
}

\author{
*Corresponding author details: Jefri Adi Kam Sitepu, M.D.; jefri_in_velilokam@yahoo.com
}

\begin{abstract}
Although tracheal stenosis is a rare consequence, it is possible in individuals undergoing extended intubation and tracheostomy. Although otorhinolaryngologists and interventional pulmonologists are often responsible for treatment, since symptoms may not manifest for weeks or months after extubation, primary care doctors are frequently the first to meet this problem. Tracheal stenosis may be mistaken for asthma in its clinical presentation, which includes dyspnea and stridor. The case described is that of a 16-year-old man with tracheal stenosis after intubation who had a series of surgical treatments to improve his airway. We analyzed common presenting symptoms, treatment choices, and preventative efforts for comparable individuals.
\end{abstract}

Keywords: tracheal stenosis; stridor; dyspnea

\section{INTRODUCTION}

Tracheal stenosis may be caused by a variety of factors, including trauma associated with surgery, intubation, or accidents, inhalation damage, or inflammatory disorders such as Wegener's granulomatosis, sarcoidosis, or systemic lupus erythematosus [1]. However, the majority of instances occur as a consequence of prolonged intubation or tracheostomy at the moment. Even with the use of a sealed high-volume, low-pressure tube, airway stenosis may develop in up to $11 \%$ of intubated or tracheostomy patients [2], even after less than 24 hours [3], [4].

When tracheal stenosis arises, definitive treatment is sometimes difficult. The most prevalent sign of tracheal stenosis is the presence of nonspecific respiratory symptoms such as wheezing, dyspnea, increasing with physical activity, stridor, and coughing, or, more often in children, a moderate respiratory tract infection resulting in acute respiratory distress [5]. These symptoms may first be misinterpreted as asthma, a far more prevalent illness, especially in children. Tracheal stenosis, on the other hand, should be evaluated if the patient with respiratory distress has a history of intubation or tracheostomy. By the time symptoms manifest, the stenosis has often reached between $30 \%$ and $50 \%$ of the lumen diameter.

Table 1 illustrates the Myer-Cotton technique for grading stenosis. The patient will develop acute respiratory distress at $80 \%$ blockage [5], [6]. This diagnosis is missed in up to $44 \%$ of patients at the time of first presentation. Many patients need emergency bronchoscopic dilatation due to the severity of the stenosis before it is correctly detected [3], [4]. Tracheal stenosis may be caused by a variety of factors, including trauma associated with surgery, intubation, or accidents, inhalation damage, or inflammatory disorders such as Wegener's granulomatosis, sarcoidosis, or systemic lupus erythematosus [1]. However, the majority of instances occur as a consequence of prolonged intubation or tracheostomy at the moment.
Even with the use of a sealed high-volume, low-pressure tube, airway stenosis may develop in up to $11 \%$ of intubated or tracheostomy patients [2], even after less than 24 hours [4], [7].

When tracheal stenosis arises, definitive treatment is sometimes difficult. The most prevalent sign of tracheal stenosis is the presence of nonspecific respiratory symptoms such as wheezing, dyspnea, increasing with physical activity, stridor, and coughing, or, more often in children, a moderate respiratory tract infection resulting in acute respiratory distress [5]. These symptoms may first be misinterpreted as asthma, a far more prevalent illness, especially in children. Tracheal stenosis, on the other hand, should be evaluated if the patient with respiratory distress has a history of intubation or tracheostomy. By the time symptoms manifest, the stenosis has often reached between $30 \%$ and $50 \%$ of the lumen diameter.

Table 1 illustrates the Myer-Cotton technique for grading stenosis. The patient will develop acute respiratory distress at $80 \%$ blockage [5], [6]. This diagnosis is missed in up to $44 \%$ of patients at the time of first presentation. Many patients need emergency bronchoscopic dilatation due to the severity of the stenosis before it is correctly detected [3], [4].

TABLE 1: Meyer-Cotton grading system [4]

\begin{tabular}{|l|l|}
\hline Grade I & $\leq 50 \%$ occlusion \\
\hline Grade II & $51-70 \%$ occlusion \\
\hline Grade III & $71-99 \%$ occlusion \\
\hline Grade IV & $100 \%$ occlusion \\
\hline
\end{tabular}




\section{CASE REPORT}

A sixteen-year-old boy was taken to the hospital for tracheal stricture repair using a customized endotracheal tube (ETT). Initially, the patient was involved in a traffic accident (crash into a power pole) in August 2016, had diminished awareness ( + ), and a tracheostomy was done to rule out extended ventilator installation. The tracheostomy was discharged in September 2016. In October 2016 (3 weeks after the tracheostomy was removed), the patient had shortness of breath as a result of a tracheal stricture at cervical vertebrae 6-7. Once again, a tracheostomy was performed. Currently, the patient is reliant on a tracheostomy, and a head CT scan reveals a narrowing of the tracheal diameter to $0.6 \mathrm{~cm}$ at the level of cervical vertebrae 6-7, with a tracheostomy tube connected at this level.
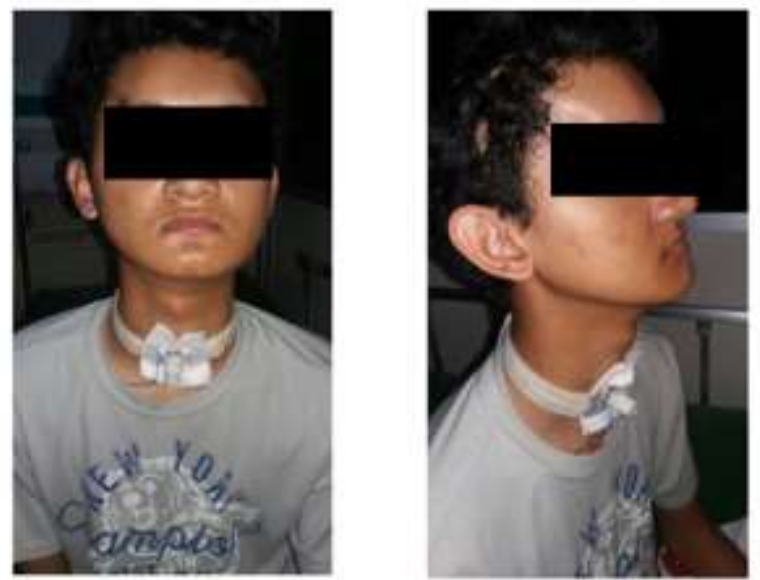

FIGURE 1: Clinical features of a 16-year-old male with a modified endotracheal tube (ETT)
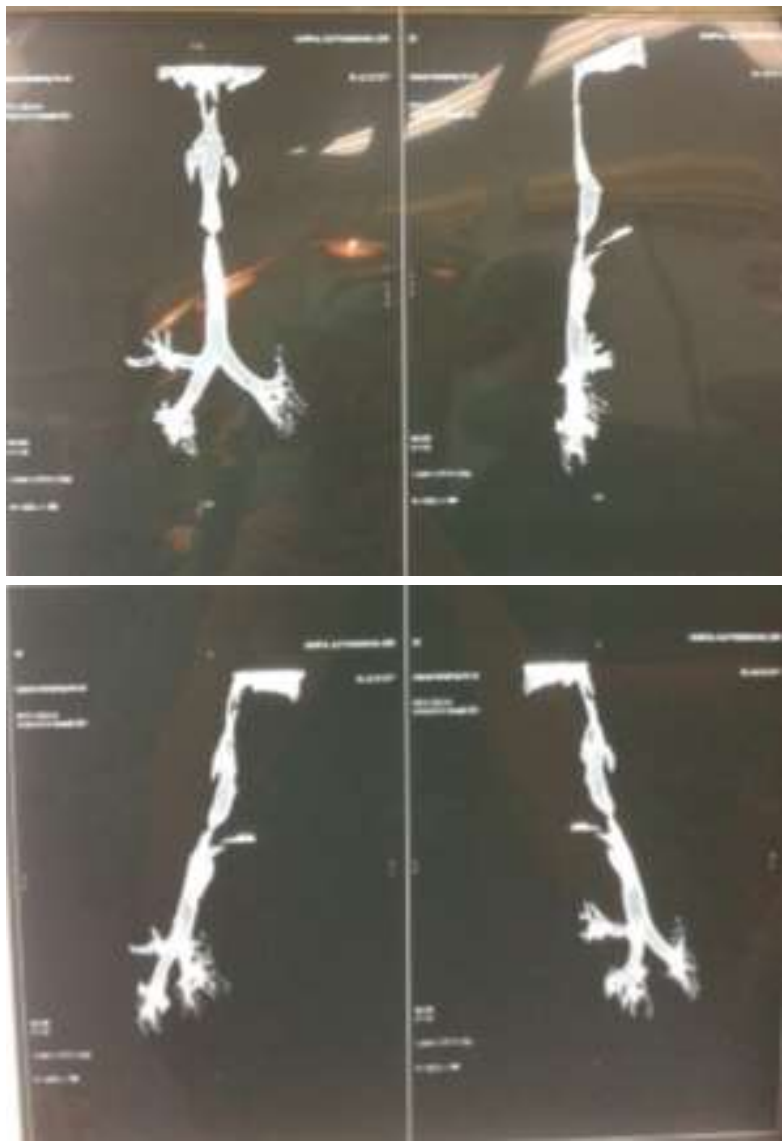

FIGURE 2: A head CT scan reveals a $0.6 \mathrm{~cm}$ narrowing of the tracheal diameter at the level of cervical vertebrae 6-7 as well as the attachment of a tracheostomy tube at the level of cervical vertebrae 7 .
The patient was then repaired using a strap muscle flap and mesh for tracheal stricture. There was a tracheal stenosis in the number 2-3 ring at the time of operation. The trachea is dislodged from the adjacent tissue. The streptococcal muscle was severed to the level of the lower tracheostomy. A vertical incision is made in the stenotic trachea to facilitate passage of the ETT tube. The tracheal defect was repaired using a strep muscle flap and a proline mesh. A sternocleidomastoid (SCM) flap was used to close the proline mesh above.

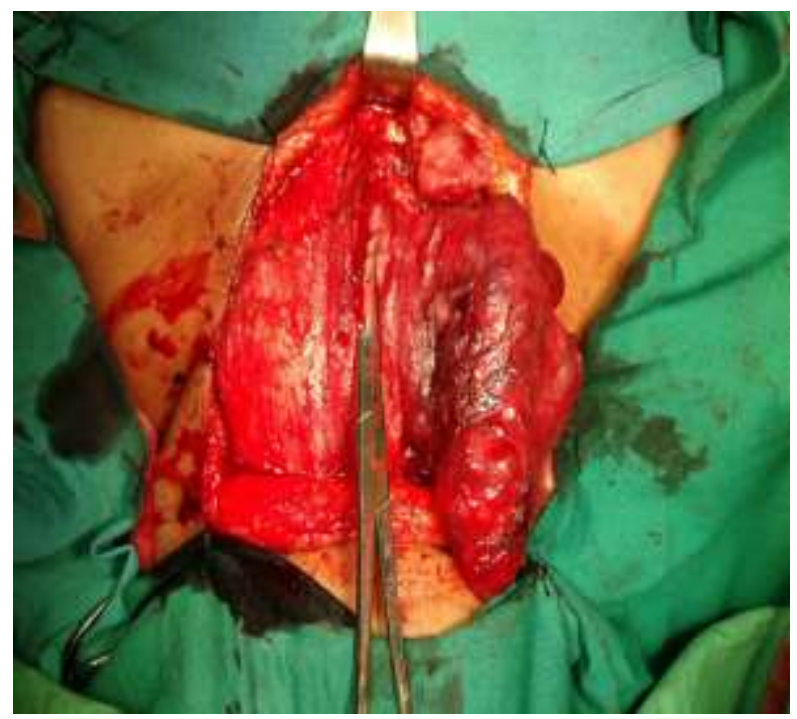

FIGURE 3: Post-repair of tracheal stenosis

\section{DISCUSSION}

Tracheal stenosis most often occurs as a consequence of prolonged intubation during which the endotracheal cuff pressure exceeds the tracheal mucosa's mean capillary pressure ( $>30 \mathrm{mmHg}$ ). Ischemia, granulation tissue, and scar tissue with luminal stricture are all consequences of excessive pressure [4], [5], [8]. This process may then result in web-like fibrosis with little or no harm to the cartilage-supporting structures. Tracheostomy-related injury is the second most prevalent cause of tracheal stenosis. These injuries can result in a variety of complications, including (1) cartilage fracture during the mechanical lift of the ventilator tube in the trachea, (2) incorrect tracheostomy tube sizing, (3) fracture during percutaneous tracheostomy tube placement, and (4) excessive granulation tissue as a result of infection and abnormal healing [9], [10].

In less than 24 hours after intubation, patients may develop tracheal stenosis [4], [7]. However, symptoms may not manifest themselves for many months after extubation with any extended intubation [2]. These criteria enhance the risk that a patient may come with dyspnea for the first time to a primary care physician. Although the invention of high-volume, low-pressure intubation cuffs significantly lowered the incidence of post-intubation stenosis, it remained around $11 \%$ in patients with a history of extended intubation [2]. This incidence may be further decreased with endotracheal treatment and meticulous stoma care, which includes recording of cuff pressure and frequent maintenance of ventilatory equipment.

Cardiovascular disease, diabetes, and illnesses requiring chronic corticosteroid usage are also common comorbidities of tracheal stenosis, all of which exacerbate microvascular damage and impair recovery [10]. Rigid bronchoscopy is the gold standard for determining the location of lesions, the diameter of the airway lumen, and the length of the stenosis. 
Computed tomography may also be used to visualize lumen constriction, which is particularly useful when the lumen is too small to accommodate the bronchoscope [4], [5].

For many years, open surgery with resection and anastomosis was considered the gold standard for almost all patients with tracheal stenosis and the only method to prevent repeated complications. Numerous recent studies have shown that the majority of patients with postintubation tracheal stenosis are unable to undergo open surgery due to severe comorbid comorbidities. Endoscopic techniques have evolved to be the most effective therapeutic choice in this instance [3], [4], [6], [8], [11]. The optimal treatment strategy should be tailored to the patient and strike a balance between a more definitive but invasive surgical resection with end-to-end anastomosis and a more conservative endoscopic approach, which bears a higher risk of recurrence.

A valuable algorithm for treatment selection is currently available, which takes into account case characteristics such as the length of the stenosis, the width of the lumen, the involvement of cartilage, and past treatment efforts [3], [8]. Current endoscopic techniques include dilation, stenting, granulation tissue removal using electrocautery, laser, or sharp incision, and the use of topical steroids or mitomycin. This is the therapy of choice for elderly individuals or those who are very difficult to open surgically. Additionally, stenotic lesions less than $20 \mathrm{~mm}$ in diameter that do not include cartilage degradation are simpler to treat endoscopically and do not need resection. Balloon dilatation or stent placement is typically the first step in endoscopic treatment; it can be combined with laser ablation of granulation tissue using a neodymiumyttrium aluminum garnet (Nd-YAG) laser and topical application of mitomycin C (MMC), which inhibits fibroblast and DNA synthesis and thus reduces granulation [2]. APC has also been utilized to decrease bleeding prior to granulation tissue excision [2]. Balloon dilatation is often repeated, sometimes three or more times, before healing occurs. When a patient has restenosis for the fourth time, resection or implantation of a stent is often contemplated for more definitive therapy [3].

Stents are associated with a plethora of additional problems, including migration, blockage, and granulation development [3], [11]. Brichet et al. [3] noted, however, that if the stent is kept in place for many months, the stenosis may grow and harden even after the stent is withdrawn. In situations with a stenosis greater than $30 \mathrm{~mm}$, cricoid injury, or loss of cartilage-supporting tissue, effective therapy is improved with open surgical resection and primary anastomosis in endoscopic care [8], [11]. Nouraei et al. [11] evaluated 62 patients with postintubation tracheal stenosis who were treated only with endoscopic procedures; the success rate decreased from $96 \%$ to $20 \%$ when the lesion exceeded 30 $\mathrm{mm}$ in length. Additionally, individuals who have suffered repeated issues after endoscopic therapy may benefit from open surgical resection and correction of the abnormality. Restenosis often develops 1-3 months after surgical resection and anastomosis [2]. This procedure has a mortality rate of around 3\% [2] and a failure rate of approximately 15\% (requiring tracheostomy tube replacement) [3], [4]. The most often seen consequences include cervicomediastinal sepsis, suture dehiscence, innominate artery rupture, aspiration pneumonia, and recurrent laryngeal nerve palsy. Grillo et al. [7] shown that using absorbable sutures nearly reduces the likelihood of granulation along the anastomosis, a typical cause of restenosis.

\section{CONCLUSION}

Patients with a history of extended intubation or tracheostomy should be assessed for post-intubation tracheal stenosis if they appear with stridor, dyspnea, cough, or wheezing. Endotracheal dilation or stenting with potential administration of mitomycin C may be a beneficial therapy option in situations with $20 \mathrm{~mm}$ stenosis and undamaged cartilage, or in individuals who are not surgical candidates. Patients with a longer and more severe stenosis (cartilage loss or recurring problems) will almost certainly need end-to-end resection and anastomosis for ultimate therapy.

\section{CONFLICTS OF INTEREST}

No competing interests declared.

\section{FUNDING}

This research received no specific grant from any funding agency.

\section{REFERENCE}

[1] N. A. Rahman, O. Fruchter, D. Shitrit, B. D. Fox, and M. R. Kramer, "Flexible bronchoscopic management of benign tracheal stenosis: Long term follow-up of 115 patients," J. Cardiothorac. Surg., vol. 5, no. 1, p. 2, 2010, doi: 10.1186/1749-8090-5-2.

[2] J. L. Wong, S. T. Tie, B. Samril, C. L. Lum, M. R. Abdul Rahman, and J. A. Abdul Rahman, "Successful treatment of tracheal stenosis by rigid bronchoscopy and topical mitomycin C: A case report," Cases Journal, vol. 3, no. 1. 2010, doi: 10.1186/1757-1626-3-2.

[3] A. Brichet et al., "Multidisciplinary approach to management of postintubation tracheal stenoses," European Respiratory Journal, vol. 13, no. 4. pp. 888893, 1999, doi: 10.1034/j.1399-3003.1999.13d32.x.

[4] S. De and S. De, "Post intubation tracheal stenosis," Indian J. Crit. Care Med., vol. 12, no. 4, pp. 194-197, 2008, doi: 10.4103/0972-5229.45081.

[5] A. S. Ho and P. J. Koltai, "Pediatric Tracheal Stenosis," Otolaryngol. Clin. North Am., vol. 41, no. 5, pp. 9991021, 2008, doi: 10.1016/j.otc.2008.04.006.

[6] L. Freitag and K. Darwiche, "Endoscopic treatment of tracheal stenosis," Thorac. Surg. Clin., vol. 24, no. 1, pp. 27-40, 2014, doi: 10.1016/j.thorsurg.2013.10.003.

[7] H. C. Grillo, D. M. Donahue, D. J. Mathisen, J. C. Wain, and C. D. Wright, "Postintubation tracheal stenosis. Treatment and results," J. Thorac. Cardiovasc. Surg., vol. 109, no. 3, pp. 486-493, 1995, doi: 10.1016/S00225223(95)70279-2.

[8] A. E. Melkane et al., "Management of postintubation tracheal stenosis: Appropriate indications make outcome differences," Respiration, vol. 79, no. 5, pp. 395-401, 2010, doi: 10.1159/000279225.

[9] A. Sarper, A. Ayten, I. Eser, A. Demircan, and E. Isin, "Review of Posttracheostomy And Postintubation Tracheal Stenosis with Special Regard to Etiology and Treatment," Internet J. Thorac. Cardiovasc. Surg., vol. 6, no. 1, 2012, doi: 10.5580/1616.

[10] N. Zias et al., "Post tracheostomy and post intubation tracheal stenosis: Report of 31 cases and review of the literature," BMC Pulm. Med., vol. 8, p. 18, 2008, doi: 10.1186/1471-2466-8-18.

[11] S. A. R. Nouraei, K. Ghufoor, A. Patel, T. Ferguson, D. J. Howard, and G. S. Sandhu, "Outcome of endoscopic treatment of adult postintubation tracheal stenosis," Laryngoscope, vol. 117, no. 6, pp. 1073-1079, 2007, doi: 10.1097/MLG.0b013e318050ca12. 